\title{
REPARATION DES PERTES DE SUBSTANCES PARTIELLES DU PAVILLON DE L'OREILLE (CONQUE) : A PROPOS DUNE OBSERVATION
}

\author{
S. KHAROUBI. \\ FACULTE DE MEDECINE DE ANNABA. ALGERIE
}

\begin{abstract}
RESUME
Les pertes de substances de la conque (pavillon de l'oreille) sont essentiellement en rapport avec l'exérèse de tumeurs malignes en particulier les carcinomes baso-cellulaires.

La reconstruction de ces defects repose sur la restauration des téguments par un lambeau rétro-auriculaire. Les résultats cosmétiques sont très satisfaisants.

Nous rapportons une observation de reconstruction du pavillon (conque) après exérèse d'un carcinome basocellulaire pigmenté par un lambeau rétro-auriculaire en deux temps.

MOTS CLES : Cancer de la peau, lambeau rétro-auriculaire, pavillon de l'oreille, reconstruction du pavillon.
\end{abstract}

The lost of concha of auricula substances are mainly due to the excision of malignant tumours, particularly baso-cellular carcinomas.

The rebuilding of these defects is based on the teguments restoration by a retro-auricular flap. The esthetic results are very satisfactory.

In this paper, we report an observation of reconstruction of the concha after excision of pigmented baso-cellular carcinoma by a retro-auricular flap made in two times.

KEY WORDS : Skin cancer, Retro-auricular flap, Pavilion of the ear, Reconstruction of the pavilion.

\section{INTRODUCTION}

Le pavillon de l'oreille par sa situation : morphologie aérienne et son siège au niveau des parois latérales du crâne est exposé aux pertes de substances traumatiques et aux tumeurs cutanées (toxicité radique).

La structure complexe du pavillon : constitution cutanéocartilagineuse et sa configuration externe : reliefs, dépression et fossettes anfractueuses rendent compte des difficultés variables de la réparation selon la zone topographique concernée.

Par ailleurs le pavillon de l'oreille constitue un élément important et primordial dans l'esthétique globale de la face et l'idendité psycho-sociale de l'individu.

La conservation ou la reconstruction d'une partie ou de la totalité du pavillon demeure donc une doléance habituelle et légitime en particulier dans le sexe féminin.

Sur la plan pratique, il est intéressant d'adopter la classification de DISANT en cinq types topographiques des pertes de substances partielles du pavillon (1).

A chaque localisation correspond une pathologie préférentielle (kératose, cancer cutané, traumatisme, plaies) et surtout un procédé adapté de réparation (suture simple, résection triangulaire et suture, lambeau d'avancement, greffe composée triangulaire).

Les pertes de substances de la conque (type V) sont le plus souvent le fait de la pathologie tumorale. Elles sont le plus souvent isolées parfois avec atteinte du méat auditif externe.
La réparation par lambeau rétro-auriculaire selon le procédé décrit par MASSON permet de pallier à ces defects avec des résultats satisfaisants (2).

\section{OBSERVATION CLINIQUE}

MR. B.L âgé de 54 ans consulte en ORL pour une lésion pigmentée du pavillon gauche évoluant depuis 10 mois.

Notre patient commerçant de profession, rapporte la notion de prurit et de «tension » sans douleurs vraies de l'oreille gauche. Des saignements épisodiques sont notés notamment après grattage ou frottement.

L'examen clinique note une tumeur bourgeonnante pigmentée de $15 \mathrm{~mm}$ dans son grand axe siégeant au niveau de la conque du pavillon de l'oreille gauche. Cette lésion est à $5 \mathrm{~mm}$ du méat acoustique externe, non transfixiante et sans infiltration des structures de voisinage. (Figure1)

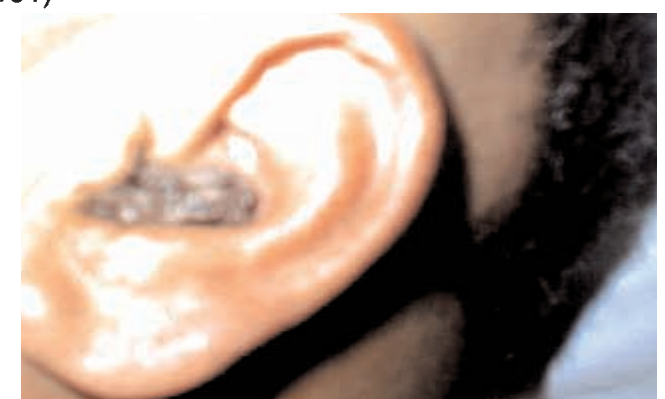

Fig. 1 : Aspect de la lésion : épithélioma baso-cellulaire pigmenté de la conque : pavillon de l'oreille gauche. 
L'otoscopie est sans particularités. II n'y a pas d'adénopathies cervicales palpables.

Sur le plan général : la peau est de phénotype brun, sans lésion de type nævi ni d'anomalies des phanères.

La biopsie de la lésion montre un épithélioma baso-cellulaire pigmenté.

Le patient demande expressément la conservation du pavillon de l'oreille. Nous lui proposons l'exérèse avec reconstruction par un lambeau cutané rétro-auriculaire en 2 temps.

Nous réalisons sous anesthésie générale une exérèse large de la lésion (emportant toute la conque) après incision d'abord hélicienne (incision horizontale au niveau du $1 / 3$ supérieur, $1 / 3$ moyen de l'hélix permettant un accès large et direct à la conque). (Figure 2)

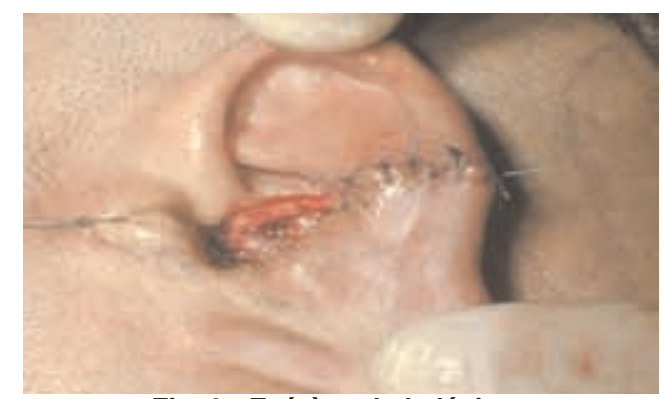

Fig. 2 : Exérèse de la lésion.

Suture de l'incision de l'hélix ayant servie pour l'abord direct de la conque.

Des recoupes sont faites au niveau du conduit auditif externe. Un lambeau cutané rétro-auriculaire à pédicule supérieur est prélevé pour réparer la perte de substance conquale. (Figure $3,4,5$ )

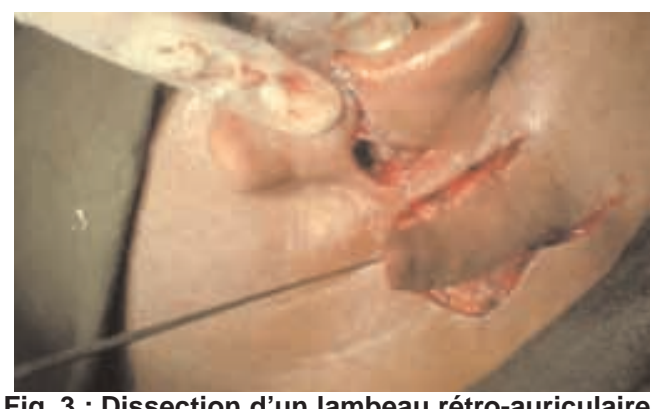

Fig. 3 : Dissection d'un lambeau rétro-auriculaire à pédicule supérieur.

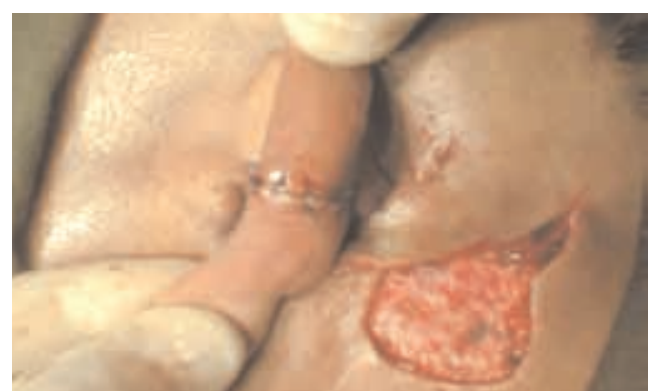

Fig. 3 : Vue postérieure : lambeau à pédicule supérieur passé en pont en direction de la perte de substance conquale.

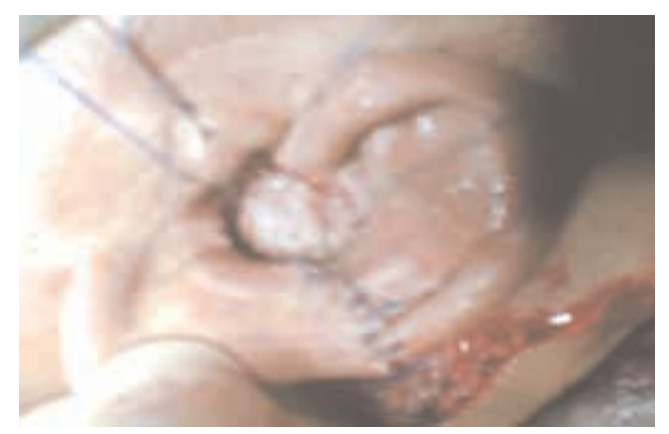

Fig. 3 : Aspect final : lambeau rétro-auriculaire comblant la perte de substance conquale.

Quinze jours plus tard le lambeau est sectionné avec aménagement du site receveur.

L'examen anatomopathologique note un épithélioma baso-cellulaire pigmenté envahissant le cartilage avec des recoupes saines.

Le résultat cosmétique est excellent. II n'y a pas de récidives, ni de sténose du conduit après 13 mois de recul.

\section{DISCUSSION}

Les pertes de substance de la région conquale sont essentiellement d'origine tumorale. Les causes traumatiques sont rares et résultent le plus souvent de l'action directe d'un agent contendant (arme blanche). Exceptionnellement, il s'agit de nécroses chondrocutanées d'origine infectieuse compliquant une otite externe ou dans les suites de chirurgie plastique (oreille décollée). Les tumeurs malignes du pavillon sont représentées principalement par les carcinomes baso-cellulaires (anciennement dénommés épithéliomas baso-cellulaires) et les carcinomes épidermoides (ou épithéliomas spino cellulaires) plus rarement par les mélanomes malins.

Le pavillon de l'oreille est très exposé aux radiations solaires d'où la fréquence des lésions pré cancéreuses (kératose actinique) et des carcinomes. II s'agit le plus souvent de sujets de sexe masculin à partir de 60 ans.

Les carcinomes baso-cellulaires sont plus fréquents au niveau du pavillon (60 à $75 \%$ ) avec une évolution essentiellement locale (3). Les carcinomes baso-cellulaires sont assez polymorphes avec néanmoins un signe pathognomonique : la perle translucide de la taille d'une tête d'épingle enchâssée dans l'épiderme. On distingue classiquement les formes planes (carcinome baso-cellulaire plan cicatriciel, sclérodermiforme, pagétoide), saillantes (nodules, bourgeons), ulcérées (ulcus rodens) ou pigmentées (carcinome baso-cellulaire tatoué : notre observation).

Ils siègent préférentiellement au niveau prétragien ou à la face postérieure du pavillon $(3,4)$.

Les carcinomes épidermoides (spino-cellulaires) sont plus agressifs avec notamment la possibilité de métastases ganglionnaires. Ils se développent surtout sur des lésions pré existantes (kératose) et siègent par ordre de fréquence au niveau de l'hélix (44 -53\%), face postérieu- 
re du pavillon (14-27\%), anthélix ou fossette triangulaire (14-19\%), sur la conque, le lobule, le tragus (5\%). La lésion est ulcérée ou ulcéro-bourgeonnante saignant au contact $(4,5)$.

Les mélanomes malins représentent $4 \%$ des cancers du pavillon et sont primitifs ou se développent sur lésions pré existantes (naevus, mélanose circonscrite de DUBREUILH). En fonction du siège on note respectivement l'atteinte préférentielle de l'hélix $(60 \%)$ puis le lobule, le tragus et la région pré tragienne, la conque et l'anthélix (3).

Le mélanome malin est de pronostic plus sévère avec une évolution locale, ganglionnaire et générale (métastases).

Le diagnostic d'une tumeur maligne du pavillon et son typage est histologique et constitue un préalable à tout projet thérapeutique.

Le carcinome baso-cellulaire réalise une prolifération monomorphe de cellules ressemblant aux cellules de l'assise basale de l'épiderme regroupées en lobules de taille variable (4).

Les formes épidermoides (carcinome spino-cellulaire) se présentent sous forme d'une prolifération lobulaire, au-delà de la membrane basale avec des cellules à différenciation épidermoide et la présence de cadres claires épi cellulaires segmentés par des desmosomes ou épines inter cellulaires (spino-cellulaire).

L'imagerie du temporal (Tomodensitométrie ou Imagerie par résonance magnétique) n'est pas systématique en dehors d'une extension particulière aux structures de voisinage (mastoïde, conduit auditif externe, parotide ou base du crâne) $(3,5)$.

La prise en charge thérapeutique des tumeurs malignes du pavillon vise à éradiquer la lésion avec des marges de résection satisfaisante (profondeur et superficie) et éviter les récidives.

Parmi les moyens thérapeutiques nous citerons les procédés non chirurgicaux ou " dermatologiques " : cryothérapie, électrocoagulation, curetage, chimiothérapie locale (fluorouracile locale) dans les formes pré cancéreuses (kératoses) ou les carcinomes très limités (3).

La curiethérapie à l'Iridium 192 est une bonne alternative dans les carcinomes du pavillon limités. Le résultat carcinologique et esthétique est corrélé à la taille de la tumeur. MAZERON et AL ont rapporté un contrôle local dans $99 \%$ avec un bon résultat cosmétique dans $78 \%$ des cas pour des carcinomes du pavillon de moins de $4 \mathrm{~cm}$ de diamètre (6).

La radiothérapie externe (électrons) est réservée aux formes étendues inopérables ; à titre de complément post opératoire (sur la lésion et les aires ganglionnaires) (7).

La chirurgie reste le traitement de base des tumeurs malignes du pavillon.

Elle comporte deux temps : l'exérèse tumorale et la réparation :

\section{L'exérèse chirurgicale}

Sous anesthésie locale (carcinomes limités) ou générale, elle doit être large 0,8 à $10 \mathrm{~mm}$ pour les carcinomes basocellulaires et épidermoides et 15 à $20 \mathrm{~mm}$ pour les mélanomes malins.

L'exérèse est le plus souvent transfixiante en raison de l'infiltration périchondro-cartilagineuse (8).

Des recoupes multiples sont nécessaires. Elles doivent être bien identifiées réalisées sur la base d'un schéma directeur. Le procédé de MOSH bien précis est intéressant mais nécessite un grand nombre de prélèvements et donc une contrainte technique évidente (9).

\section{La réparation}

Une suture par rapprochement est possible en cas d'exérèse limitée. Une cicatrisation dirigée par des soins locaux (pansements gras) est parfois retenue dans certaines localisations ; face postérieure du pavillon, région pré tragienne ou lobule. Une exérèse étendue et surtout transfixiante impose le recours aux procédés de reconstructions du pavillon $(1,10)$.

Plusieurs procédés de réparation sont possibles pour les pertes de substances partielles du pavillon. Ils dépendent essentiellement du siège de ce defect (1).

La reconstruction des pertes de substances de la conque (type $V$ ) sont avantageusement traités par les lambeaux rétro-auriculaires (11). En effet seul le recouvrement tégumentaire pose problème ; le support cartilagineux n'est pas nécessaire à ce niveau (site habituel de prélèvement cartilagineux pour les rhinoplasties ou la chirurgie de l'oreille moyenne).

Le lambeau rétro-auriculaire à pédicule supérieur ou inférieur est suturé en avant et latéralement au niveau de la perte de substance conquale (12). Le pédicule est sectionné 15 à 21 jours plus tard le plus souvent sous anesthésie locale avec aménagement du site receveur.

Une autre variante technique consiste à désépidermisé le lambeau rétro-auriculaire (après sa levée) sur un segment de $10 \mathrm{~mm}$ environ correspondant au passage sous et rétro-antéhélicien. L'absence de cartilage à ce niveau évite la compression du pédicule désépidermisé lors de la bascule antérieure du lambeau (2).

Ce procédé a l'avantage, en plus de sa simplicité, d'une réalisation en un temps.

Les résultats cosmétiques des reconstructions isolées de la conque par lambeau rétro-auriculaire sont satisfaisants. Des complications sont possibles :

- infection locale avec risque de périchondrite par propagation au reste du pavillon dont la prévention passe par une asepsie rigoureuse, la qualité des soins post opératoires et l'antibiothérapie systématique,

- hématomes,

- nécrose partielle ou totale secondaire à la compression du lambeau dans son trajet sous et rétro-auriculaire.

II peut y avoir également des problèmes de cicatrisation : rétraction du lambeau, cicatrice chéloïde ou troubles de la pigmentation locale. 
Les tumeurs étendues et évoluées, récidivantes ou compliquées de périchondrite avec nécrose cartilagineuse et rétraction du pavilion, peuvent relever des procédés de reconstruction ou otopoièse.

Ces procédés sont complexes nécessitent souvent plusieurs temps opératoires et font appel à différents greffons: fascia superficialis temporalis, autogreffon cartilagineux, greffe de peau libre. Les résultats cosmétiques restent très moyens voir médiocres.

Le recours à une épithèse de pavillon après mise en place de fixtures ostéo-intégrées pour amarrage de la prothèse nous semble une alternative intéressante car plus simple, régulièrement efficace et ses résultats sur le plan cosmétique sont très satisfaisants (13).

\section{CONCLUSION}

Les pertes de substance de la conque sont le plus souvent le fait de la pathologie tumorale : carcinome basocellulaire, épidermoide ou plus rarement un mélanome malin.

Les impératifs de la chirurgie carcinologique doivent être largement satisfait avec résection " généreuse " et à distance. L'exérèse radicale sans récidives reste l'objectif prioritaire.
On se trouve souvent confronté à une demande d'un protocole de conservation du pavillon.

Le recours aux procédés de reconstruction par lambeau rétro-auriculaire permet de satisfaire cette doléance avec des résultats cosmétiques intéressants.

L'alternative à ces problèmes reste le dépistage et le traitement précoce de ces lésions tumorales eu utilisant les procédés de réparation peu invasifs (suture simple, cicatrisation dirigée).

\section{REFERENCES}

1- Disant F., Muller P. Traumatismes et tumeurs du pavillon de l'oreille Procédés de reconstruction. Encyclopédie Médico-Chirurgicale ( Elsevier Paris) ORL. 1994 ; 46-101 $10 \mathrm{P}$.

2- Masson J.K.. A simple island flap for reconstruction of concha-helix defects Br J Plast Surg. 1972; 25 : 399-403

3- Toquet J., Malard O., Beauvillain De Montreuilc. Tumeurs malignes de l'oreille Encyclopédie Médico-Chirurgicale ( Elsevier - Paris) ORL 1999 20247 A10 10 P.

4- Dubertret L. Cancers cutanés Paris Flammarion Médecine-Sciences 1992. 5- Beauvillain De Montreuil C., Ferri-launay M.L., Peron P. et al Tumeurs primitives étendues de l'oreille IN: TRAISSAC. L EDT : les cancers de l'oreille XXVII congrès de la société française de carcinologie cervico-faciale. Paris Masson 1995 1-6.

6- Mazeron JJ, Baillet F., Le Bourgeois J.P Curiethérapie du pavillon de l'oreille IN: TRAISSAC. L EDT : les cancers d l'oreille

XXVII congrès de la société française de carcinologie cervico-faciale. Paris Masson 1995 114-120.

7- Hoffstetter F., Guillemin F., Allavena C. et al Carcinomes du pavillon de l'oreille externe traités au centre alexis-vautrin.IN: TRAISSAC. L EDT : les cancers d l'oreille XXVII congrès de la société française de carcinologie cervico-faciale. Paris Masson 1995 121-127.

8- Texier M., Preaux J. Reconstruction partielle de l'oreille IN Banzet P,
Servant JM Chirurgie Plastique Reconstructrice Esthétique

Paris: Médecines- Sciences Flammarion 1994 : $357-360$.

9- Mohs. FE , Larson P. , Iriondo M. Micrographic surgery for the microscopically controlled excision of carcinoma of the external ear. J Am Acad Dermatol 1988; $19: 729-737$

10- Revol M , Servant JM. Reconstruction du pavillon de l'oreille Manuel de chirurgie plastique reconstructrice et réparatrice Paris Editions PRADEL 1993.

11- Mouly R., Papadopoulos P., De Taddeo P. Lambeau sous cutané pour la reconstruction de la conque et de la face antérieure du pavillon de l'oreille. Ann Chiru Plast1979; $24: 191-194$.

12- Gordon SD « button » skin repair of an ear detect Plast Reconstr Surg $1971 ; 48: 190$.

13- Tjellstrom A, Rosenhall N, Lindstrom J. et al Five years experience with skin penetrating bone anchored implants in the temporal bone Acta Otolaryngol 1993; $95:$ 568-575. 